\title{
Bedeutung der Formeln.
}

(Aufgefürt sind nur Formeln, die in vorliegendem Werke verwendet sind, und aucb nur insoweit, als eine Erklärung nicht in demselben Artikel gegeben ist. Die Aufzählung erfolgt rein alphabetisch, und zwar entsprecbend der im Auskunftsbuch gebrauchten Schreibung, dabei aufsteigend nach dem zugebörigen Index, so dass $z$. $\mathrm{B}$. $\mathrm{CH}_{4}$ vor $\mathrm{C}_{\mathbf{2}} \mathrm{H}_{2} \mathrm{O}_{4}$, dieses vor $\mathrm{C}_{3} \mathrm{H}_{6}$, dieses wieder vor $\mathrm{C}_{5} \mathrm{H}_{6} \mathrm{~N}$ zu suchen ist.)

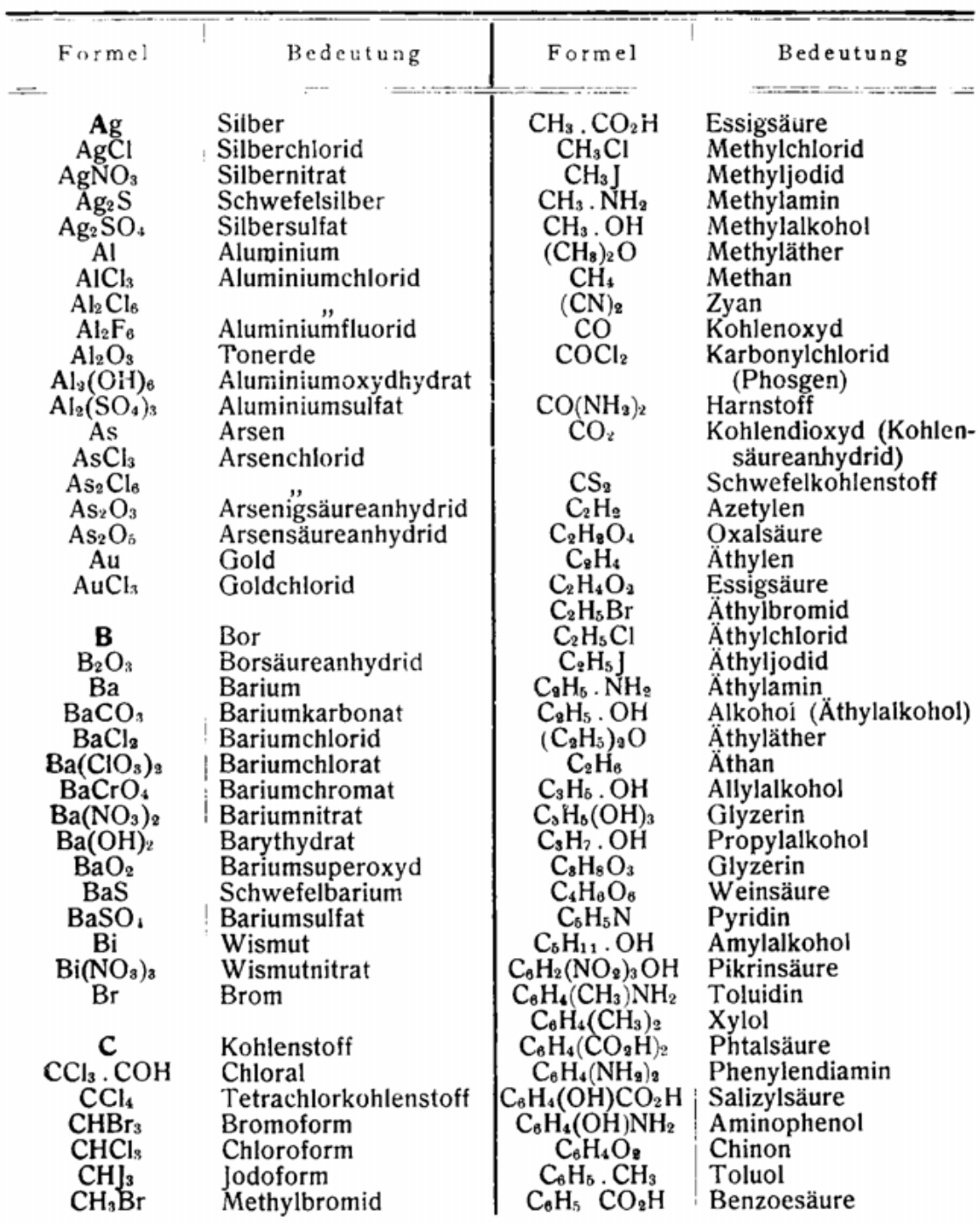




\begin{tabular}{|c|c|c|c|}
\hline Formel & Bedeutung & Formel & Bedeutung \\
\hline 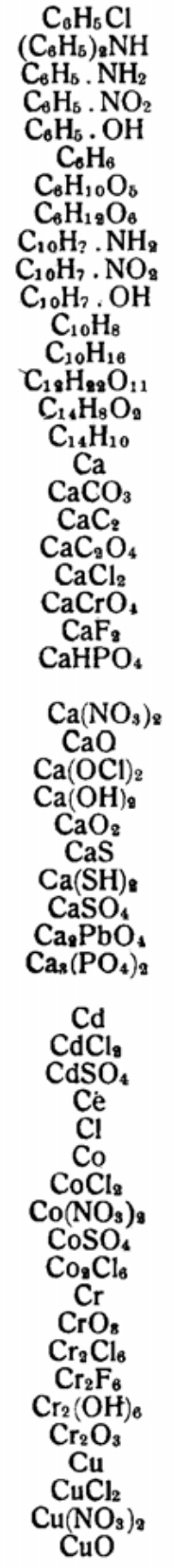 & $\begin{array}{l}\text { Chlorbenzol } \\
\text { Diphenylamin } \\
\text { Anilin } \\
\text { Nitrobenzol } \\
\text { Phenol (Karbolsäure) } \\
\text { Benzol } \\
\text { Zellulose, Stärke } \\
\text { Traubenzucker } \\
\text { Naphthylamin } \\
\text { Nitronaphtalin } \\
\text { Naphtol } \\
\text { Naphtalin } \\
\text { Terpen } \\
\text { Rohrzucker } \\
\text { Anthrachinon } \\
\text { Anthrazen } \\
\text { Kalzium } \\
\text { Kalziumkarbonat } \\
\text { Kalziumkarbid } \\
\text { Kalziumoxalat } \\
\text { Kalziumchlorid } \\
\text { Kalziumchromat } \\
\text { Kalziumfluorid } \\
\text { sekundär. Kalzium- } \\
\text { phosphat } \\
\text { Kalziumnitrat } \\
\text { Kalziumoxyd (Kalk) } \\
\text { Kalziumhypochlorit } \\
\text { Kalziumhydrat } \\
\text { Kalziumsuperoxyd } \\
\text { Kalziumsulfid } \\
\text { Kalziumsulfhydrat } \\
\text { Kalziumsulfat } \\
\text { Kalziumplumbat } \\
\text { tertiäres Kalzium- } \\
\text { phosphat } \\
\text { Kadmium } \\
\text { Kadmiumchlorid } \\
\text { Kadmiumsulfat } \\
\text { Zer } \\
\text { Chlor } \\
\text { Kobalt } \\
\text { Kobaltchlorür } \\
\text { Kobaltnitrat } \\
\text { Kobaltsulfat } \\
\text { Kobaltchlorid } \\
\text { Chrom } \\
\text { Chromsäureanhydrid } \\
\text { Chromchlorid } \\
\text { Chromfluorid } \\
\text { Chromhydroxyd } \\
\text { Chromoxyd } \\
\text { Kupfer } \\
\text { Kupferchlorid } \\
\text { Kupfernitrat } \\
\text { Kupferoxyd } \\
\text { Kal }\end{array}$ & 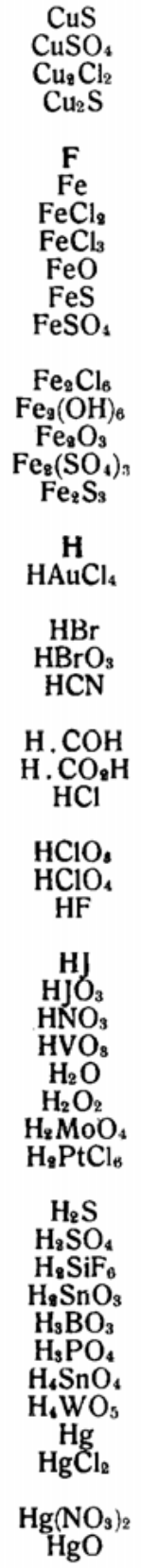 & 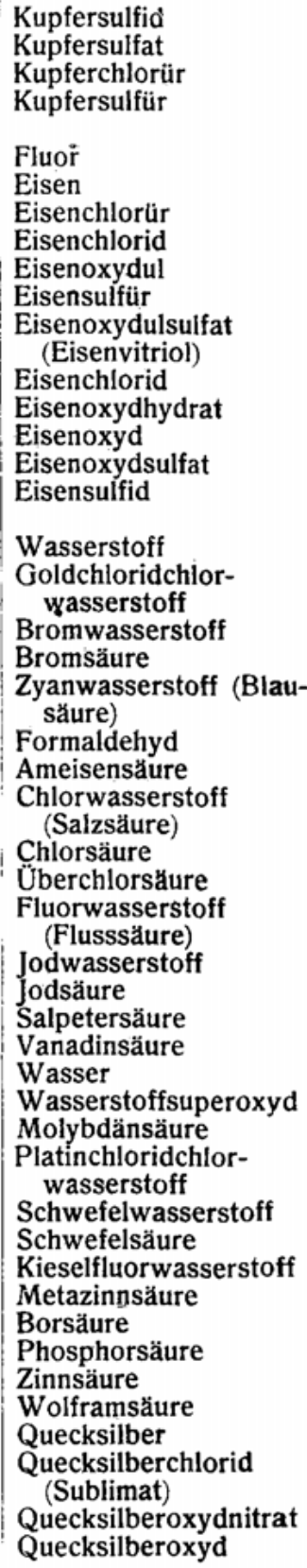 \\
\hline
\end{tabular}




\begin{tabular}{|c|c|c|c|}
\hline Formel & Bcdeutung & Formel & Bedeutung \\
\hline$=-1=$ & & & $=1-1$ \\
\hline $\mathrm{HgS}$ & Schwefelquecksilber & $\mathrm{Mg}\left(\mathrm{NO}_{\mathbf{s}}\right)_{2}$ & Magnesiun \\
\hline $\mathrm{HgS̆O}_{4}$ & $\mathrm{Qu}$ & Mg & \\
\hline & $\begin{array}{l}\text { Quec } \\
\text { (Ko }\end{array}$ & $\mathrm{Mg}(\mathrm{OH})_{2}$ & xydhydrat \\
\hline $\mathrm{Hg}_{2}\left(\mathrm{NO}_{3}\right)_{2}$ & $\begin{array}{l}\text { Quecksilberoxydul- } \\
\text { nitrat }\end{array}$ & $\underset{\mathrm{Mn}}{\mathrm{MgSO}_{4}}$ & $\begin{array}{l}\text { Magnesiumsulfat } \\
\text { Mangan }\end{array}$ \\
\hline & & $\mathrm{MnCO}_{3}$ & Mar \\
\hline $\mathbf{J}$ & Jod & $\mathrm{MgC}$ & Mas \\
\hline $\mathbf{J} \mathbf{r}$ & Iridium & $\mathrm{MnO}_{2}$ & $\begin{array}{l}\text { ansuperoxyd } \\
\text { ansulfat }\end{array}$ \\
\hline$\underset{\mathrm{KBr}}{\mathbf{K}}$ & Kalium & $\mathrm{Mo}^{4}$ & Molybdän \\
\hline $\mathrm{KBrO}_{3}$ & Kaliu & $\mathbf{N}$ & Stickstoff \\
\hline $\mathrm{KC}_{2} \mathrm{H}_{2} \mathrm{O}_{2}$ & Kaliur & $\left(\mathrm{NH}_{2}\right)_{2}$ & \\
\hline $\mathrm{KC}_{4} \mathrm{H}_{5} \mathrm{O}_{6}$ & $\begin{array}{l}\text { bitartrat (Wein- } \\
\text { 1) }\end{array}$ & $\underset{\mathrm{NH}_{3}}{\mathrm{NH}_{2} \cdot \mathrm{OH}}$ & $\operatorname{lamin}_{\mathrm{k}}$ \\
\hline $\mathrm{KCN}$ & Zyank & $\mathrm{NH}_{4} \mathrm{Br}$ & Nat \\
\hline KCNS & & $\mathrm{NH}_{4} . \mathrm{C}$ & $\mathrm{Am}$ \\
\hline $\begin{array}{c}\mathrm{KClO}_{3} \\
\mathrm{KCl}_{3}\end{array}$ & $\begin{array}{l}\text { Kalit } \\
\text { Kalit }\end{array}$ & $\mathrm{NH}$ & $\begin{array}{l}\text { Ammoniumchlorid } \\
\text { (Salmiak) }\end{array}$ \\
\hline$\underset{\mathrm{KF}}{\mathrm{KClO}_{4}}$ & $\begin{array}{l}\text { Kaliump } \\
\text { Kaliumf }\end{array}$ & $\begin{array}{l}\mathrm{NH}_{4} \mathrm{ClO}_{3} \\
\mathrm{NH}_{4} \mathrm{ClO}_{4}\end{array}$ & $\begin{array}{l}\text { liumchlorat } \\
\text { iumperchlorat }\end{array}$ \\
\hline $\mathrm{KHCO}_{3}$ & Kalit & $\left(\mathrm{NH}_{4}\right)_{2} \mathrm{CrO}_{4}$ & omat \\
\hline $\mathrm{KHSO}_{3}$ & & $\left(\mathrm{NH}_{4}\right)_{8} \mathrm{Cr}_{2} \mathrm{O}_{7}$ & $\begin{array}{l}\text { hromat } \\
\text { fhydrat }\end{array}$ \\
\hline $\mathrm{KJ}$ & Kali & $\left(\mathrm{NH}_{4}\right)_{2} \mathrm{HPO}$ & sphat \\
\hline$\underset{\mathrm{KNO}_{2}}{\mathrm{KMnO}_{4}}$ & anganat & $\begin{array}{c}\mathrm{NH}_{4} \mathrm{~J} \\
\mathrm{NH}_{4} \mathrm{NO}_{3}\end{array}$ & Am \\
\hline & t (Kali- & $\begin{array}{c}\left(\mathrm{NH}_{4}\right)_{2} \mathrm{SO}_{4} \\
\mathrm{NO}\end{array}$ & fat \\
\hline $\mathrm{KOH}$ & at (Ätzkali) & & Stickstoffo \\
\hline & & & ul \\
\hline $\mathrm{K}_{2} \mathrm{C}_{2} \mathrm{O}_{4}$ & Kalit & & Stickstoffdiox \\
\hline $\begin{array}{l}\mathrm{K}_{2} \mathrm{C}_{4} \mathrm{H}_{4} \mathrm{O}_{3} \\
\mathrm{~K}_{2} \mathrm{CrO}_{4}\end{array}$ & & $\begin{array}{c}\mathrm{N}_{2} \mathrm{O}_{6} \\
\mathrm{Na}\end{array}$ & ersäureanhydrid \\
\hline $\mathrm{K}_{2} \mathrm{Cr}_{2} \mathrm{O}_{7}$ & $\mathrm{~K}$ & & \\
\hline $\begin{array}{c}\mathrm{K}_{2} \mathrm{O} \\
\mathrm{K}_{2} \mathrm{Pt}(\mathrm{CN})_{4}\end{array}$ & $\begin{array}{l}\text { Kalit } \\
\text { Kalit }\end{array}$ & & $\begin{array}{l}\text { Natr } \\
\text { Natr }\end{array}$ \\
\hline $\mathrm{K}_{2} \mathrm{PtCl}_{8}$ & $\begin{array}{l}\text { Kaliumplatinchlorid } \\
\text { Kaliumsulfid }\end{array}$ & $\begin{array}{l}\mathrm{NaC}_{2} \mathrm{H}_{3} \mathrm{O}_{2} \\
\mathrm{NaCl}\end{array}$ & $\begin{array}{l}\text { Natriumazetat } \\
\text { Natriumchlorid (Koch- }\end{array}$ \\
\hline & Kalit & $\mathrm{NaClO}_{3}$ & Natriumchlorat \\
\hline $\mathrm{K}_{2} \mathrm{SO}_{4}, \mathrm{Kl}_{2} \mathrm{SiF}_{6}\left(\mathrm{SO}_{4}\right)_{3}$ & $\begin{array}{l}\text { Kalialaun } \\
\text { Kieselfluornatrium }\end{array}$ & $\underset{\mathrm{NaF}}{\mathrm{NaClO}_{4}}$ & $\begin{array}{l}\text { Natriumperchlorat } \\
\text { Natriumfluorid }\end{array}$ \\
\hline $\begin{array}{l}\mathrm{K}_{2} \mathrm{SiO}_{3} \\
\mathrm{~K}_{3} \mathrm{PO}_{4}\end{array}$ & $\begin{array}{l}\text { Kaliumsilikat } \\
\text { Kaliumphosphat }\end{array}$ & $\begin{array}{l}\mathrm{NaHCO}_{3} \\
\mathrm{NaHSO}_{3}\end{array}$ & $\begin{array}{l}\text { Natriumbika } \\
\text { Natriumbisu }\end{array}$ \\
\hline $\mathbf{L i}$ & Lith & $\mathrm{NaH}_{2} \mathrm{PO}_{4}$ & primär. Natrium- \\
\hline $\mathrm{LiCl}$ & Lithiumchlorid & & \\
\hline $\begin{array}{l}\mathrm{Li}_{2} \mathrm{CO}_{3} \\
\mathrm{Li}_{2} \mathrm{SO}_{4}\end{array}$ & $\begin{array}{l}\text { Lithiumkarbonat } \\
\text { Lithiumsulfat }\end{array}$ & $\begin{array}{r}\mathrm{Na} \\
\mathrm{NaMr}\end{array}$ & $\begin{array}{l}\text { Natriumjodid } \\
\text { Natriumpermanganat }\end{array}$ \\
\hline $\mathbf{M g}$ & Magn & & Natriumnitrit \\
\hline$\underset{\mathrm{MgCl}_{2}}{\mathrm{MgCO}_{3}}$ & $\begin{array}{l}\text { Magnesiumkarbonat } \\
\text { Magnesiumchlorid }\end{array}$ & & $\begin{array}{l}\text { Natriumnitrat (Chili- } \\
\text { salpeter) }\end{array}$ \\
\hline
\end{tabular}




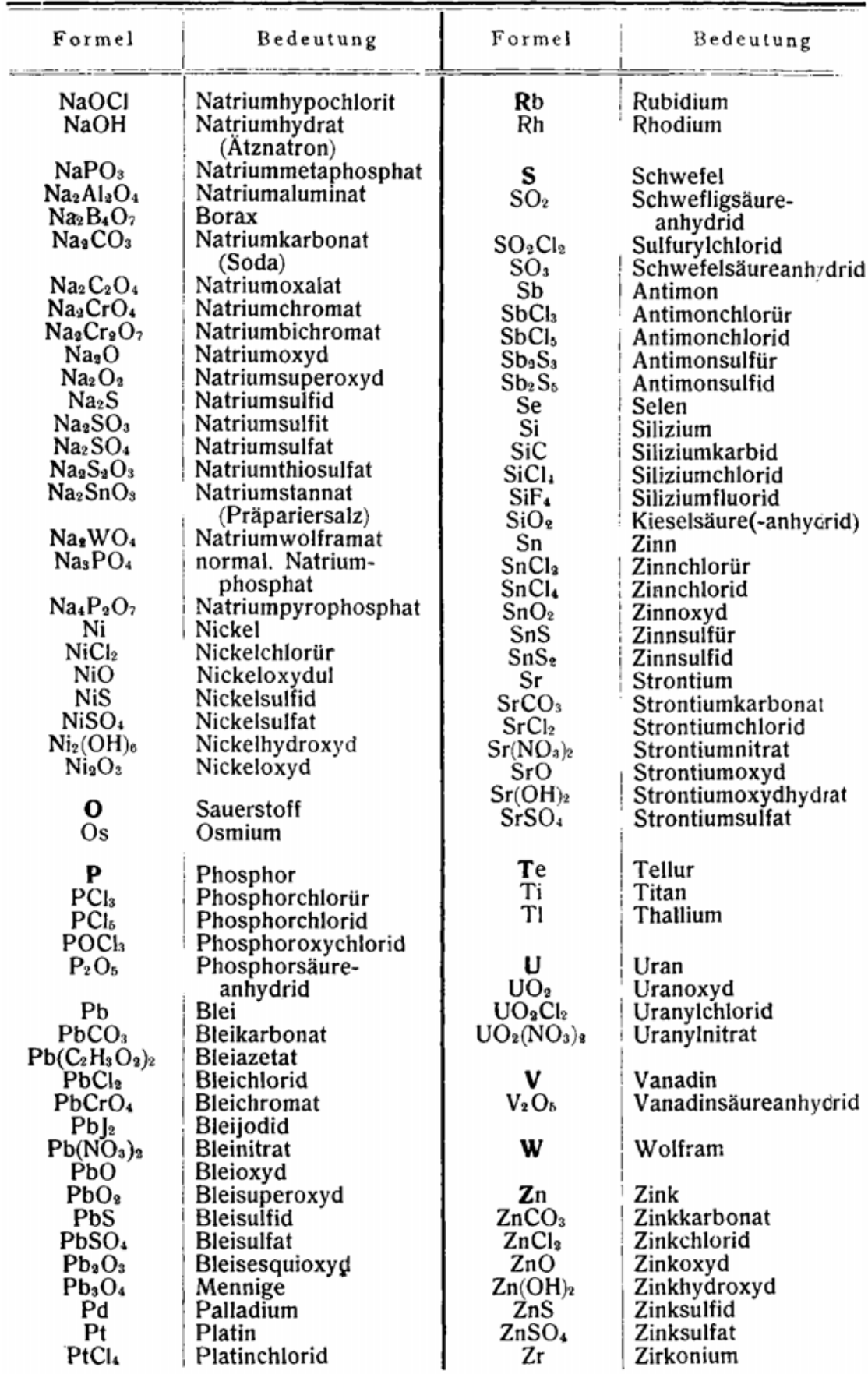

